Pacific

Journal of

Mathematics

GYROGROUPS AND THE DECOMPOSITION OF GROUPS INTO TWISTED SUBGROUPS AND SUBGROUPS

Tuval Foguel and Abraham A. Ungar 


\title{
GYROGROUPS AND THE DECOMPOSITION OF GROUPS INTO TWISTED SUBGROUPS AND SUBGROUPS
}

\author{
Tuval Foguel and Abraham A. Ungar
}

\begin{abstract}
Gyrogroups are generalized groups modelled on the Einstein groupoid of all relativistically admissible velocities with their Einstein's velocity addition as a binary operation. Einstein's gyrogroup fails to form a group since it is nonassociative. The breakdown of associativity in the Einstein addition does not result in loss of mathematical regularity owing to the presence of the relativistic effect known as the Thomas precession which, by abstraction, becomes an automorphism called the Thomas gyration. The Thomas gyration turns out to be the missing link that gives rise to analogies shared by gyrogroups and groups. In particular, it gives rise to the gyroassociative and the gyrocommuttive laws that Einstein's addition possesses, in full analogy with the associative and the commutative laws that vector addition possesses in a vector space. The existence of striking analogies shared by gyrogroups and groups implies the existence of a general theory which underlies the theories of groups and gyrogroups and unifies them with respect to their central features. Accordingly, our goal is to construct finite and infinite gyrogroups, both gyrocommutative and non-gyrocommutaive, in order to demonstrate that gyrogroups abound in group theory of which they form an integral part.
\end{abstract}

\section{Introduction.}

A gyrogroup is a grouplike structure that is defined in [7] along with a weaker structure called a left gyrogroup. We show that any given group can be turned into a left gyrogroup which is, in turn, a gyrogroup if and only if the given group is central by a 2-Engel group. The importance of left gyrogroups stems from the facts that (i) any gyrotransversal groupoid is a left gyrogroup, and (ii) any left gyrogroup is a twisted subgroup in a specified group.

Twisted subgroups are subsets of groups, introduced by Aschbacher [1], which under general conditions are near subgroups. The concept of near subgroup of a finite group was introduced by Feder and Vardi [6] as a tool to study problems in computational complexity involving the class $N P$. 
In a previous article [7] we have shown that every gyrogroup is a twisted subgroup in some specified group, and introduced the involutory decomposition of a group $G=B H$ into a twisted subgroup $B$ and subgroup $H$, demonstrating that the resulting twisted subgroups are gyrocommutative gyrogroups. Gyrogroups are grouplike objects which share analogies with groups. In particular, gyrogroups are classified into gyrocommutative and non-gyrocommutative ones in full analogy with groups, thus offering a broader context for the study of groups. Gyrogroups are structures which first arose in the study of Einstein's relativistic velocity addition law. Gyrogroup theory captures the mathematical regularity that has seemingly been lost in the transition from ordinary vector addition (which is a group operation) to Einstein's relativistic velocity addition (which, being nonassociative, is not a group operation).

Following the involutory decomposition that we presented in [7], we present in this article a non-involutory decomposition of groups into twisted subgroups and subgroups. The resulting twisted subgroups, in this case, turn out to be gyrogroups that need not be gyrocommutative.

In Section 2 we show that any left gyrogroup is a gyrotransversal of some group. In Section 3, we show that any group can be turned into the diagonal transversal of a specified group. Hence, by Theorem 3.4, any group can be turned into a left gyrogroup. We then characterize in Theorem 3.7 the groups that their associated left gyrogroups are gyrogroups. Finally, two examples of nongyrocommutative gyrogroups, one finite and one infinite, are presented in Section 4.

\section{Left gyrogroups as gyrotransversals of groups.}

The definitions and many of the theorems and lemmas refered to in this paper appear in [7].

Definition 2.1. A transversal groupoid $(B, \odot)$ of a subgroup $H$ in a group $G$ is a gyrotransversal of $H$ in $G$ if

(i) $1_{G} \in B, 1_{G}$ being the identity element of $G$;

(ii) $B=B^{-1}$; and

(iii) $B$ is normalized by $H, H \subseteq N_{G}(B)$, that is, $h B h^{-1} \subseteq B$ for all $h \in H$.

Definition 2.2 (Gyrosemidirect Product Groups). Let $P=(P, \odot)$ be a left gyrogroup, and let $\operatorname{Aut}_{o}(P)=\operatorname{Aut}_{o}(P, \odot)$ be any subgroup (not necessarily the smallest one) of $\operatorname{Aut}(P, \odot)$ containing all the gyroautomorphisms of $(P, \odot)$. The gyrosemidirect product group

$$
P \rtimes \operatorname{Aut}_{o}(P)
$$

of the left gyrogroup $P$ and a gyroautomorphism group $\operatorname{Aut}_{o}(P)$ is a group of pairs $(x, X)$, where $x \in P$ and $X \in \operatorname{Aut}_{o}(P)$, with group operation given 
by the gyrosemidirect product

$$
(x, X)(y, Y)=(x \odot X y, \operatorname{gyr}[x, X y] X Y) .
$$

It is anticipated in Definition 2.2 that the gyrosemidirect product group (2.1) is a group with group operation given by the gyrosemidirect product (2.2). This is indeed the case as we show in the following:

Lemma 2.3. Let $(P, \odot)$ be a left gyrogroup, and let $\operatorname{Aut}_{o}(P, \odot)$ be any of its gyroautomorphism groups. Then the gyrosemidirect product $P \rtimes_{।} \operatorname{Aut}_{o}(P)$ is a group.

Proof. We view the elements $(x, X)$ of $P \times \operatorname{Aut}_{o}(P)$ as bijections of $P$ according to the equation

$$
(x, X) g=x \odot X g
$$

for any $x, g \in P$ and $X \in \operatorname{Aut}_{o}(P)$, where the identity bijection is the pair $(1, I)$, and the inverse of $(x, X)$ is $(x, X)^{-1}=\left(\left(X^{-1} x\right)^{-1}, X^{-1}\right)$, thus the elements of $P \times \operatorname{Aut}_{o}(P)$ indeed act bijectively on $P$. To show that the elements of $P \times \operatorname{Aut}_{o}(P)$ are a subgroup of the symmetric group on $P$ we employ the left cancellation law, which is valid in any left gyrogroup. As such, the set $P \times \operatorname{Aut}_{o}(P)$ forms a group of bijections with group operation given by composition,

$$
\begin{aligned}
(x, X)(y, Y) g & =(x, X)(y \odot Y g) \\
& =x \odot X(y \odot Y g) \\
& =x \odot(X y \odot X Y g) \\
& =(x \odot X y) \odot \operatorname{gyr}[x, X y] X Y g \\
& =(x \odot X y, \operatorname{gyr}[x, X y] X Y) g .
\end{aligned}
$$

The composition turns out to be the gyrosemidirect product in $P \rtimes_{\triangleleft} \operatorname{Aut}_{o}(P)$ thus verifying that the operation in the gyrosemidirect product group is indeed a group operation.

Theorem 2.4. Every left gyrogroup $P=(P, \odot)$ is a gyrotransversal of its gyroautomorphism group $\operatorname{Aut}_{o}(P)$ in its gyrosemi-direct product group $P \rtimes_{1}$ $\operatorname{Aut}_{o}(P)$.

Proof. Let $H=\operatorname{Aut}_{o}(P)$ be any gyroautomorphism group of the left gyrogroup $(P, \odot)$, and let $G=P \rtimes_{\mid} H$ be the resulting gyrosemidirect product group. Identifying elements $h \in H$ with pairs $(1, h) \in G, H$ is isomorphic with a subgroup of $G$. Similarly, identifying elements $p \in P$ with their corresponding pairs $\left(p, 1_{H}\right) \in G, P$ is a subset of $G$. The left gyrogroup $P$ and the two groups $H$ and $G$ share their identity elements which are respectively denoted by $1_{P}, 1_{H}$ and $1_{G}$. We note that in any left gyrogroup $(P, \odot)$ the gyroautomorphisms gyr $\left[p, 1_{P}\right]$ and gyr $\left[1_{P}, p\right]$ are the identity automorphism of $P$, as shown in [23]. 
The left gyrogroup $P$, considered as a set, is the transversal of $H$ in $G=$ $P \rtimes_{1} H$ by Definition 2.2. In order to show that $(P, \odot)$ is a gyrotransversal of $H$ in $G$ it remains to verify that the transversal operation in $P$ equals its left gyrogroup operation $\odot$, and establish the validity of properties (i), (ii) and (iii) in Definition 2.1 of gyrotransversals.

Let $\left(x, 1_{H}\right),\left(y, 1_{H}\right) \in P \subset G$ be any two elements of $P$. Their product in $G$, Definition 2.2, has the unique decomposition

$$
\begin{aligned}
\left(x, 1_{H}\right)\left(y, 1_{H}\right) & =(x \odot y, \operatorname{gyr}[x, y]) \\
& =\left(x \odot y, 1_{H}\right)\left(1_{P}, \operatorname{gyr}[x, y]\right)
\end{aligned}
$$

indicating that the transversal binary operation in $P$ is the gyrogroup operation, $\odot$, in $P$.

Clearly, $P$ contains the identity element $\left(1_{P}, 1_{H}\right)$ of $G$. Moreover, since in $G,\left(p, 1_{H}\right)^{-1}=\left(p^{-1}, 1_{H}\right)$ for any $p \in P$, we have $\left(p, 1_{H}\right)^{-1} \in P$ so that $P=P^{-1}$, thus establishing properties (i) and (ii) of Definition 2.1.

To show that $H$ normalizes $P$ in $G$, property (iii) in Definition 2.1, we note that for all $p \in P$ and $h \in H$ we have by Definition 2.2,

$$
\begin{aligned}
\left(1_{P}, h\right)\left(p, 1_{H}\right)\left(1_{P}, h^{-1}\right) & =\left(1_{P} \odot p^{h}, \operatorname{gyr}\left[1_{P}, p^{h}\right] h 1_{H}\right)\left(1_{P}, h^{-1}\right) \\
& =\left(p^{h}, h\right)\left(1_{P}, h^{-1}\right) \\
& =\left(p^{h} \odot 1_{P}^{h}, \operatorname{gyr}\left[p^{h}, 1_{P}^{h}\right] h h^{-1}\right) \\
& =\left(p^{h}, 1_{H}\right) \in P
\end{aligned}
$$

and the proof is thus complete.

\section{Diagonal Transversals.}

Definition 3.1 (Diagonal Transversals). Let $K$ be a group and let $G=$ $K \rtimes \operatorname{Inn}(K)$ be the semidirect product group of $K$ and $\operatorname{Inn}(K)$, where $\operatorname{Inn}(K)$ is the inner automorphism group of $K$ whose generic element $\alpha_{k}$ denotes conjugation by $k \in K$. Then, the diagonal transversal $D$ generated by $K$ (in $G$ ) is a subset of $G$ given by

$$
D=\left\{\left(k, \alpha_{k}\right) \mid k \in K\right\} \subset G .
$$

Any element $\left(k, \alpha_{k}\right) \in D$ is determined by a corresponding element $k \in K$. We therefore use the notation

$$
D(k)=\left(k, \alpha_{k}\right)
$$

to denote the elements of $D$.

We may note that if $K$ is commutative then $\operatorname{Inn}(K)=\left\{1_{K}\right\}$ is the trivial group, and the transversal of $\operatorname{Inn}(K)$ in $G$ is isomorphic with the group $G$.

It is anticipated in Definition 3.1 that diagonal transversals are transversals. In the following theorem we will show that this is indeed the case. In 
fact, we will show that diagonal transversals are special transversals; they are gyrotransversals.

Theorem 3.2. A diagonal transversal with its transversal operation, is a gyrotransversal groupoid.

Proof. Let $D$ be a diagonal transversal generated by a group $K$, and let $G=K \rtimes \operatorname{Inn}(K)$. Any element $\left(k, \alpha_{h}\right)$ of $G$, where $k, h \in K$, can be uniquely written as a product

$$
\left(k, \alpha_{h}\right)=\left(k, \alpha_{k}\right)\left(1_{K}, \alpha_{k^{-1} h}\right)=D(k)\left(1_{K}, \alpha_{k^{-1} h}\right)
$$

of an element in $D$ and an element in $\operatorname{Inn}(K)$. Hence, $D$ is a transversal of $\operatorname{Inn}(K)$ in $G$.

In order to find the transversal operation $\odot$ of the transversal $D$ let us consider the product decomposition

$$
\left(k_{1}, \alpha_{k_{1}}\right)\left(k_{2}, \alpha_{k_{2}}\right)=\left(k_{1} k_{2}^{k_{1}}, \alpha_{k_{1} k_{2}}\right)=\left(k_{1} k_{2}^{k_{1}}, \alpha_{k_{1} k_{2}^{k_{1}}}\right)\left(1_{K}, \alpha_{\left(k_{2}^{-1}\right)^{k_{1} k_{2}}}\right)
$$

which can be written as

$$
D\left(k_{1}\right) D\left(k_{2}\right)=D\left(k_{1} k_{2}^{k_{1}}\right)\left(1_{K}, \alpha_{\left[k_{1}, k_{2}^{-1}\right]}\right)
$$

for any $k_{1}, k_{2} \in K$, where $\left[k_{1}, k_{2}\right]=k_{1} k_{2} k_{1}^{-1} k_{2}^{-1}$ is the commutator of $k_{1}$ and $k_{2}[\mathbf{1 5}$, p. 92]. It follows from the product decomposition (3.2) that the transversal operation is given by

$$
D\left(k_{1}\right) \odot D\left(k_{2}\right)=D\left(k_{1} k_{2}^{k_{1}}\right) .
$$

As a byproduct we also find from the product decomposition (3.2) that the element $h \in \operatorname{Inn}(K) \subset G$ determined by any two elements $D\left(k_{1}\right)$ and $D\left(k_{2}\right)$ of the transversal $D$, Definition 2.8 of [7], is

$$
h\left(D\left(k_{1}\right), D\left(k_{2}\right)\right)=\left(1_{K}, \alpha_{\left[k_{1}, k_{2}^{-1}\right]}\right) \in \operatorname{Inn}(K)
$$

$h$ being the transversal map $D \times D \rightarrow \operatorname{Inn}(K)$. To show that the transversal groupoid $(D, \odot)$ is a gyrotransversal groupoid, we will verify the validity of properties (i)-(iii) in Definition 2.1.

(i) The identity $\left(1_{K}, \alpha_{1_{K}}\right)$ of $G$ is the identity element $D\left(1_{K}\right)$ in $D$.

(ii) In $D, D\left(k_{1}\right)^{-1}=D\left(k^{-1}\right)$ so $D=D^{-1}$.

(iii) $\operatorname{Inn}(K) \subseteq N_{G}(D)$ since

$$
\begin{aligned}
\left(1_{K}, \alpha_{h}\right) D(k)\left(1_{K}, \alpha_{h^{-1}}\right) & =\left(1_{K}, \alpha_{h}\right)\left(k, \alpha_{k}\right)\left(1_{K}, \alpha_{h^{-1}}\right) \\
& =\left(k^{h}, \alpha_{k^{h}}\right)=D\left(k^{h}\right) \in D
\end{aligned}
$$

and the proof is complete. 
Remark 3.3. Let $K$ be a group, let $G=K \rtimes \operatorname{Inn}(K)$ be the semidirect product group of $K$ and $\operatorname{Inn}(K)$, and let $D$ be the diagonal transversal formed by $K$ in $G$. Then, $D$ is also a gyrotransversal in the group $D \rtimes_{1}$ $\operatorname{Inn}(K)$. Since, $\operatorname{Inn}(K)$ contains all the gyrations of the left gyrogroup $D$.

Following Definition 3.1, any group $(K, \cdot)$ generates a diagonal transversal

$$
D=\left\{D(k)=\left(k, \alpha_{k}\right) \mid k \in K\right\}
$$

whose elements $D(k) \in D$ are identified with the elements $k \in K$. By Theorem 3.2, the diagonal transversal $D$ forms a gyrotransversal groupoid whose binary operation is given by Eq. (3.3), and whose gyrations are derived from Eq. (3.4) by means of Eq. (2.2) of [7]. By Theorem 2.13 of [7], any gyrotransversal groupoid is a left gyrogroup. Under the obvious identification of the set $D$ of the left gyrogroup $(D, \odot)$ with the set $K$ of the group $(K, \cdot)$, this group is turned into the left gyrogroup $(K, \odot)$

(i) where the left gyrogroup operation is given by

$$
a \odot b=a b^{a}
$$

as we see from Eq. (3.3), and

(ii) when the gyrations of $K$ are the automorphisms

$$
\left.\operatorname{gyr}[a, b]=\alpha_{\left[a, b^{-1}\right.}\right]
$$

of $K$ as we see from Eq. (3.4). We have proved the following theorem:

Theorem 3.4. Any group $(K, \cdot)$ can be turned into an associated left gyrogroup $(K, \odot)$ by introducing into $(K, \cdot)$ the left gyrogroup operation $\odot$ which is given in terms of the group operation . by

$$
a \odot b=a b^{a} .
$$

The gyroautomorphisms of the resulting left gyrogroup $(K, \odot)$ are given by the equation

$$
\left.\operatorname{gyr}[a, b]=\alpha_{\left[a, b^{-1}\right.}\right]
$$

for all $a, b \in K$.

Definition 3.5 (Associated Left Gyrogroups). The associated left gyrogroup of a group $(K, \cdot)$ is the left gyrogroup $(K, \odot)$ whose left gyrogroup operation $\odot$ is given in terms of the group operation $\cdot$ by $a \odot b=a b^{a}$ for all $a, b \in K$.

Theorem 3.6. The associated left gyrogroup $(K, \odot)$ of a group $(K, \cdot)$ is a group if and only if $(K, \cdot)$ is nilpotent of class 2 .

Proof. By Definition 2.3 of [7] the left gyrogroup $(K, \odot)$ is a group if and only if $\operatorname{gyr}[a, b]=I$ is the identity automorphism of $K$ for all $a, b \in K$. But, by Eq. (3.7), $\operatorname{gyr}\left[a, b^{-1}\right]=I$ for all $a, b \in K$ if and only if $\alpha_{[a, b]}=I$ for all $a, b \in K$. The latter is equivalent to the condition $[a, b] \in Z(K)$ for all 
$a, b \in K$, that is, the condition that $K$ is nilpotent of class 2 is equivalent to the condition that $(K, \odot)$ is a group, as desired.

A 2-Engel group $E$ is a group satisfying $[[a, b], b]=1$. Engel groups are useful in various studies of nilpotency; see e.g. [14]. It becomes evident from the following Theorem that these are useful in the study of gyrogroups as well.

Theorem 3.7. Let $(K, \odot)$ be the left gyrogroup associated with a group $(K, \cdot)$. Then $(K, \odot)$ is a gyrogroup if and only if $(K, \cdot)$ is central by a 2Engel group.

Proof. A left gyrogroup is a gyrogroup if and only if it possesses the loop property, by Definition 2.3 of [7]. We thus have to characterize the loop property identity

$$
\operatorname{gyr}[a \odot b, b]=\operatorname{gyr}[a, b]
$$

in $(K, \odot)$ in terms of a characterization of 2-Engel groups. Identity (3.10) in $(K, \odot)$ is equivalent to the identity

$$
\alpha_{\left[a b^{a}, b^{-1}\right]}=\alpha_{\left[a, b^{-1}\right]}
$$

in $(K, \cdot)$. Identity $(3.11)$, viewed in $(K, \cdot) / Z(K)$, is equivalent to the identity

$$
\left[a b^{a}, b^{-1}\right]=\left[a, b^{-1}\right]
$$

in $(K, \cdot) / Z(K)$. This, in turn, is equivalent to the more revealing identity

$$
[a, b]=\left[b^{-1}, a\right]
$$

in $(K, \cdot) / Z(K)$, as we see from the following chain of equivalent identities.

$$
\begin{aligned}
{\left[a b^{a}, b^{-1}\right]=\left[a, b^{-1}\right] } & \Leftrightarrow a^{2} b a^{-1} b^{-1} a b^{-1} a^{-2} b=a b^{-1} a^{-1} b \\
& \Leftrightarrow b^{-1}=a b a^{-1} b^{-1} a b^{-1} a^{-1} \\
& \Leftrightarrow 1=a b a^{-1} b a b^{-1} a^{-1} b^{-1} \\
& \Leftrightarrow[[a, b], b]=1 .
\end{aligned}
$$

Finally, the identity (3.14) characterizes the 2-Engel groups [14]. Hence, the loop property identity (3.11) in the group $(K, \cdot)$ is equivalent to the identity (3.14) in the quotient group $(K, \cdot) / Z(K)$. The latter, in turn, is equivalent to the condition that $(K, \cdot) / Z(K)$ is a 2-Engel group, as desired.

Corollary 3.8. The left gyrogroup $(K, \odot)$ associated with any nilpotent group $(K, \cdot)$ of class 3 is a gyrogroup.

Proof. Any nilpotent group of class 3 is central by a 2-Engel group [14]. The result thus follows from Theorem 3.7. 
Following Corollary 3.8, gyrogroup formalism can be applied to nilpotent groups of class 3 (or, more generally, to any group which is central by a 2-Engel group). Gyrogroup formalism, proved useful in the special theory of relativity and in hyperbolic geometry [23], is presently being found useful in group theory as well.

As an example of the application of gyrogroup formalism to nilpotent groups of class 3 , given

$$
x \odot a=b
$$

then there exists a unique solution for $x$,

$$
x=b \odot(\operatorname{gyr}[b, a] a)^{-1}
$$

in the abstract gyrogroup $(G, \odot)$ by an equation and its solution in a nilpotent group $(K, \cdot)$ of class 3 . Let $(K, \odot)$ be the gyrogroup generated by $(K, \cdot)$ according to Definition 3.5.

According to Eq. (3.8), Equation $(3.15)$ in $(K, \odot)$ takes the form

$$
x a^{x}=b
$$

in $(K, \cdot)$, and according to both Eqs. (3.9) and (3.8) its unique solution $(3.16)$ in $(K, \odot)$ takes in $(K, \cdot)$ the form

$$
\begin{aligned}
x & =b \odot\left(a^{\left[b, a^{-1}\right]}\right)^{-1} \\
& =b \odot\left(a^{-1}\right)^{\left[b, a^{-1}\right]} \\
& =b\left(\left(a^{-1}\right)^{\left[b, a^{-1}\right]}\right)^{b} \\
& =b\left(a^{-1}\right)^{b\left[b, a^{-1}\right]} .
\end{aligned}
$$

By means of gyrogroup theory we have thus obtained in the following Corollary a group theoretic identity that would be very tedious to obtain otherwise.

Corollary 3.9. Let $G$ be a group which is central by a 2-Engel group. Then the equation

$$
x a^{x}=b
$$

for $x$ in $G$ possesses the unique solution

$$
x=b\left(a^{-1}\right)^{b\left[b, a^{-1}\right]} .
$$

Remark 3.10. It can readily be shown by induction on the nilpotency class that Equation $(3.17)$ for the unknown $x$ in any nilpotent group $(G, \cdot)$ possesses a unique solution. It is true when $(G, \cdot)$ is abelian, and an induction on the class (divide out the center) does the rest. Hence, for any nilpotent group $(G, \cdot)$ the associated groupoid $(G, \odot)$ is a left gyrogroup that forms a loop. Consequently, there are loops which are left gyrogroups but are not gyrogroups. 
Remark 3.11. Any loop $(L, *)$ has an associativity correction $C$ satisfying $a *(b * c)=(a * b) * C[a, b] c$ for all $a, b \in L$. The loop $L$ is a left gyrogroup if and only if $C[a, b]$ is an automorphism of $L$ for any $a, b \in L$. Examples of loops which are non-gyrogroup left gyrogroups are provided in Remark 3.10 .

\section{Examples.}

Example 4.1. The lowest order of non-group gyrogroups generated from nilpotent groups of class 3 which are not of class 2 is 16 . Using the software package MAGMA and its library [4] we found three nonisomorphic nilpotent groups of order 16 which are of class 3 but are not of class 2 (see Theorem 3.6). They generate three non-gyrocommutative gyrogroups of order 16 , denoted by $K_{16}, L_{16}$, and $M_{16}$. The gyrogroup multiplication table of one of them, $K_{16}$, Can be calculated by employing Eq. (3.8). The gyroautomorphisms of $K_{16}$ can be calculated by means of Eq. (3.9). There is only one non-identity gyroautomorphism $A$. The set of all gyroautomorphisms of $K_{16},\{I, A\}$, is a group of order 2. In general, the set of all gyroautomorphisms of a gyrogroup need not form a group. Thus, for instance, the gyroautomorphisms of the Einstein 2-dimensional gyrogroup $\left(\Re_{c}^{2}, \oplus_{E}\right)$ are all rotations of the Euclidean plane $\Re^{2}$ about its origin, but there is no gyroautomorphism that rotates the plane about its origin by $\pi$ radians [21]. $K_{16}$ contains a group $H$ which is a normal subgroup of the gyrogroup $K_{16}$ (see Definitions 4.7 and 4.8 in [7]). The quotient gyrogroup $K_{16} / H$ turns out to be an abelian group. Hence, we have in hand an example of an extension of a group by another group that gives a nonassociative structure (that is, the gyrogroup $K_{16}$ ). It is an extension which is far from being trivial, where $H$ and $K_{16} / H$ are groups while $K_{16}$ is a non-gyrocommutative gyrogroup.

Example 4.2 (A non-group, non-gyrocommutative matrix gyrogroup). The matrix group $\left(T_{4}, \cdot\right)$ of all $4 \times 4$ real or complex upper triangular matrices with diagonal elements 1 ,

$$
M(x)=\left(\begin{array}{cccc}
1 & x_{1} & x_{2} & x_{3} \\
0 & 1 & x_{4} & x_{5} \\
0 & 0 & 1 & x_{6} \\
0 & 0 & 0 & 1
\end{array}\right)
$$

under matrix multiplication is nilpotent of nilpotency class 3. Hence, by Corollary 3.8 the pair $\left(T_{4}, \odot\right)$ is a gyrogroup whose gyrogroup operation is given by

$$
M(x) \odot M(y)=(M(x))^{2} M(y)(M(x))^{-1} .
$$

The gyroautomorphisms gyr $[M(a), M(b)]$ of the gyrogroup $\left(T_{4}, \odot\right)$ are given in terms of their effects on $M(z)$ by the equation

$$
\operatorname{gyr}[M(a), M(b)] M(z)=(M(a) \odot M(b))^{-1} \odot(M(a) \odot(M(b) \odot M(z)))
$$


for all $M(a), M(b), M(z) \in\left(T_{4}, \odot\right)$.

Acknowledgments. We have been assisted by Jeremy Dover of NSA in the use of the software package MAGMA [4]. We wish to thank him, and Michael Kinyon of Indiana University South Bend for helpful suggestions about the paper. During a seminar that Joseph Rotman gave about the present paper at the University of Illinois, Sergei Ivanov made Remark 3.10, thus providing us with examples of left gyrogroups which are loops but fail to be gyrogroups. This and other useful remarks made during the seminar were e-mailed to us by Joseph Rotman. We are pleased to acknowledge financial support fron ND EPSCoR grants \#OSR-9452892 (awarded to TF) and \#OSE-9452892 (awarded to AU).

\section{References}

[1] Michael Aschbacher, Near subgroups of finite groups, J. Group Theory, 1 (1998), 113-129.

[2] Nicolas Bourbaki, Algebra, Addison-Wesley, 1974.

[3] Richard H. Bruck, A Survey of Binary Systems, Springer-Verlag, 1966.

[4] J. Cannon and C. Playoust, An introduction to MAGMA, University of Sydney, Sydney, 1993.

[5] O. Chein, Hala O. Pflugfelder and Jonathan D.H. Smith (eds.), Quasigroups and Loops Theory and Applications, Sigma Series in Pure Mathematics, Vol. 8, Heldermann Verlag, Berlin, 1990.

[6] T. Feder and M. Vardi, The computational structure of monotone monadic SNP and constraint satisfaction: A study through Datalog and group theory, SIAM J. Comput., 28(1) (1999), 57-104.

[7] Tuval Foguel and Abraham A. Ungar, Involutory decomposition of groups into twisted subgroups and subgroups, J. Group Theory, 3(1) (2000), 27-46.

[8] Tomas Kepka and Jon D. Phillips, Connected transversals to subnormal subgroups, Comm. Math. Univ. Carolinae, 38 (1997), 223-230.

[9] Markku Niemenmaa, On multiplication groups of loops, J. Algebra, 135 (1990), 112122.

[10] (1996), 135-142.

[11] Markku Niemenmaa and Tomas Kepka, On connected transversals to Abelian subgroups in finite groups, Bull. London Math. Soc., 24 (1992), 343-346.

[12] Markku Niemenmaa and Ari Vesanen, On connected transversals in the projective special linear group PSL(2,7), J. Algebra, 166 (1994), 455-460.

[13] Hala O. Pflugfelder, Quasigroups and Loops: Introduction, Heldermann Verlag, Berlin, 1990.

[14] Derek J.S. Robinson, A Course in the Theory of Groups, Springer, New York, 1995.

[15] Joseph J. Rotman, An Introduction to the Theory of Groups, Springer, New York, 1995. 
[16] Lev V. Sabinin, On the equivalence of categories of loops and homogeneous spaces, Soviet Math. Dokl., 13 (1972), 970-974.

[17] Abraham A. Ungar, Thomas rotation and the parametrization of the Lorentz transformation group, Found. Phys. Lett., 1 (1988), 57-89.

[18] - Axiomatic approach to the nonassociative group of relativistic velocities, Found. Phys. Lett., 2 (1989), 199-203.

[19] - The relativistic noncommutative nonassociative group of velocities and the Thomas rotation, Results Math., 16 (1989), 168-179.

[20] _ Weakly associative groups, Results Math., 17 (1990), 149-168.

[21] _ Thomas precession and its associated grouplike structure, Amer. J. Phys., 59 (1991), 824-834.

[22] _ The holomorphic automorphism group of the complex disk, Aequat. Math., 47 (1994), 240-254.

[23] - Thomas precession: Its underlying gyrogroup axioms and their use in hyperbolic geometry and relativistic physics, Found. Phys., 27 (1997), 881-951.

[24] - From Pythagoras to Einstein: The hyperbolic Pythagorean theorem, Found. Phys., 28 (1998), 1283-1321.

Received April 21, 1999 and revised June 4, 1999.

North Dakota State University

FARGO, ND 58105

E-mail address: tuval_foguel@ndsu.nodak.edu

North Dakota State University

FARGO, ND 58105

E-mail address: ungar@ndsu.Nodak.edu 